\title{
Determinantes para um Sistema de Informação Florestal
}

\author{
Humberto Angelo ${ }^{1}$, Alexandre Nascimento de Almeida ${ }^{2}$, \\ Paulo José Prudente de Fontes ${ }^{3}$, Álvaro Nogueira de Souza ${ }^{1}$ \\ ${ }^{1}$ Faculdade de Engenharia Florestal, Universidade de Brasília - UnB, Brasília/DF, Brasil \\ ${ }^{2}$ Faculdade UnB de Planaltina - FUP, Universidade de Brasília - UnB, Planaltina/DF, Brasil \\ ${ }^{3}$ Laboratório de Produtos Florestais, Instituto Brasileiro do Meio Ambiente e dos Recursos Naturais Renováveis - IBAMA, \\ Brasília/DF, Brasil
}

\begin{abstract}
RESUMO
Este trabalho testa a importância relativa de um grupo de temas pré-selecionados, contribuindo para a construção de um Sistema de Informações Florestais para o Brasil - SIFLOR-BR. A metodologia empregada se pauta em um conjunto de 12 módulos temáticos e 58 subtemas para serem avaliados por um grupo de pessoas (método de juízes holístico). As informações referem: 1) Cobertura florestal; 2) Florestas de produção;3) Propriedade das terras florestais; 4) Indústria de base florestal; 5) Mercado de produtos florestais; 6) Tecnologia de produtos; 7) Biodiversidade florestal; 8) Mudanças florestais e climáticas; 9) Economia e estatística florestal; 10) Legislação florestal; 11) Certificação florestal; 12) Ensino e pesquisa florestal. Conclui-se que as principais informações que deve conter o SIFLOR concentram-se nos temas Produção e Economia florestal.
\end{abstract}

Palavras-chave: sistema de informações florestais, administração florestal, setor florestal.

\section{Determinants for a Forest Information System}

\begin{abstract}
Determinants for a forest information system. This paper tests the relative importance of a group of pre-selected themes for the construction of a Forestry Information System for Brazil - BR-SIFLOR. The methodology that was used lies on a set of 12 thematic modules and 58 sub-themes in order to be evaluated by a group of people (holistic judging method). The information relates to: 1) Forest cover; 2) Production Forests; 3) Ownership of Forest Lands; 4) Forest-based Industry; 5) Forest Products Market; 6) Technology Products; 7) Forest Biological Diversity; 8) Climate Change and Forest; 9) Forest Economics and Statistics; 10) Forest Law; 11) Forest Certification; 12) Forestry Teaching and Research. We conclude that the key information to be included in the SIFLOR focus on themes of Forest Economy and Production.
\end{abstract}

Keywords: forest information system, forest management, forest sector.

\section{INTRODUÇÃO}

A importância do Brasil no contexto mundial florestal não pode ser ignorada, mais da metade do território nacional é composto de florestas, ou seja,
509,8 milhões de hectares são cobertos por florestas nativas e 6,8 milhões de hectares por florestas plantadas, classificando o país como a segunda maior área florestal 
da Terra, superada apenas pela Rússia, com 809 milhões de hectares com florestas (SFB, 2010).

As florestas naturais brasileiras, principalmente a Floresta Amazônica, pela sua extensão, abrigam a maior biodiversidade do planeta e protegem a quinta parte da água doce disponível na Terra (Brasil, 2005). Apesar da importância desse extenso patrimônio florestal e da relevância do setor para a economia do país, existe, ainda, uma carência de ferramentas e instrumentos que ajudem a planejar e realizar gestão florestal de forma eficaz. Essa deficiência afeta também o processo de análise e avaliação desse potencial e, consequentemente, a decisão dos gestores públicos e privados quanto à utilização, de forma sustentável, dos recursos florestais brasileiros.

Tentativas na proposição de um Sistema de Informações (SI) na área florestal remontam à década de 1970, com o trabalho de Beattie \& Nascimento (1979). Posteriormente, Brena (1995) e Graça et al. (2002) também abordaram essa questão. Desses autores emana o consenso da necessidade de se implementar um Sistema de Informações Florestais para o país em razão da importância ambiental, econômica e social que os recursos florestais têm para os diversos segmentos estratégicos da economia brasileira.

O desenvolvimento do setor florestal no Brasil necessita de instrumentos de conhecimento permanente das realidades florestais, os quais possam auxiliar no planejamento estratégico do uso racional dos recursos e, também, ajudar na sua conservação. Além de subsídio para o planejamento e a gestão de florestas públicas, esse tipo de ferramenta contribui para que o setor público atue como agente de desenvolvimento do setor florestal e não somente como executor de políticas de ações de comando e controle.

O governo federal aprovou a lei n. 11.284, de 2/3/2006, que trata da gestão de florestas públicas para a produção sustentável e cria o Serviço Florestal Brasileiro. No entanto, para uma implantação adequada desse serviço, há necessidade de que ele seja alimentado com informações básicas e estratégicas para o planejamento, a coordenação e a execução da gestão de florestas públicas.

No contexto da gestão pública, Soares \& Quintella (2005) afirmam que a Tecnologia da Informação (TI) deixou de ter papel restrito de suporte administrativo, passando a participar na aplicação estratégica de tomadas de decisão, implementação e avaliação de políticas governamentais. De acordo com Rezende (2004), governantes e gestores públicos têm enfrentado mudanças substanciais nas formas de conduzir a governança pública, considerando a necessidade de ações práticas, dinâmicas e inteligentes, para as quais informações oportunas e conhecimentos personalizados são fundamentais.

Para Albertin (1996), a implementação de um Sistema de Informação (SI) significa uma mudança, muitas vezes profunda, na organização, que deve ser planejada e preparada para garantir o seu sucesso. De uma maneira geral, os SI podem ser classificados em operacionais, gerenciais e estratégicos (Laudon \& Laudon, 2004). Do ponto de vista do seu gerenciamento, os SI podem ser conceituados como uma combinação estruturada de informação, recursos humanos, TI e práticas de trabalho, organizados de forma a permitir o melhor atendimento aos objetivos de uma organização (Campos, 1994).

De acordo com Lachtermacher (2002), converter dados brutos (números e fatos) em informações gerenciais para serem utilizadas no processo de tomada de decisão é o que se espera de um SI. Embora muitos autores reconheçam que SI corresponde a algo mais abrangente do que os sistemas de informação computadorizados, a grande maioria discute apenas os sistemas computadorizados. A pesquisa em SI vem procurando identificar e estudar os inúmeros fatores que afetam o uso de computadores e sistemas de informação nas organizações (Davis, 1989; Rodrigues \& Ludmer, 2005).

Um sistema de informações florestais que aborde aspectos relacionados à produção e à comercialização de produtos florestais constitui a base para a definição de estratégias e para a tomada de decisões. Segundo Nobre et al. (2003), os sistemas de informações florestais não evoluíram na mesma velocidade dos sistemas industriais e administrativos.

O planejamento de um Sistema de Informações Florestais deve definir um conjunto de ações visando elaborar a concepção do sistema e a execução de um modelo para atender aos objetivos propostos (Beattie \& Nascimento, 1979; Brena, 1995; Graça et al., 2002).

Assim sendo, o trabalho aborda a construção de um Sistema de Informações Florestais para o Brasil (SIFLOR-BR). Em especial, busca identificar as variáveis e os módulos temáticos desse sistema. 


\section{MATERIAL E MÉTODOS}

\subsection{Abrangência do estudo}

Esta pesquisa abrangeu recursos florestais naturais e plantados localizados nos diferentes biomas brasileiros e relacionados como as atividades de gestão florestal e do setor de base florestal considerando: acesso ao recurso florestal, sistemas de produção com base no manejo florestal, indústria florestal, comércio e mercado de produtos florestais madeireiros e não madeireiros, indicadores socioeconômicos, estatísticas florestais, certificação, biodiversidade florestal, pesquisa e serviços ambientais.

Selecionaram-se, inicialmente, temas ou módulos temáticos que agregavam os principais tipos de informações florestais, como base nos trabalhos de: Beattie \& Nascimento (1979) e Brena (1995). A Tabela 1 apresenta a proposta inicial dos 58 principais tipos de informação florestal agrupados em 12 módulos temáticos.

\subsection{Método de juízes}

A proposta inicial com os módulos temáticos e os tipos de informações principais (Tabela 1) passou por um processo de validação. Utilizou-se o método de juízes holístico que visa submeter uma proposta à apreciação de um grupo de experts, denominados juízes, com o objetivo de que eles possam julgar a pertinência ou não da proposta (Vasques et al., 2011; Silva \& Almeida, 2009).

Foi enviado um questionário com a referida proposta para um grupo selecionado de 160 juízes, distribuídos em 4 subgrupos de 40 juízes, representando os segmentos: a) setor público (gestores públicos, analistas ambientais e técnicos de governo); b) setor privado (empresários, gerentes, técnicos e representantes de associações de classe do setor de base florestal); c) setor acadêmico

Tabela 1. Módulos temáticos e os principais tipos de informações florestais.

Table 1. Thematic modules and the main types of forest information.

\begin{tabular}{|c|c|}
\hline $\begin{array}{l}\text { MÓDULO } \\
\text { TEMÁTICO }\end{array}$ & PRINCIPAIS TIPOS DE INFORMAÇÕES FLORESTAIS \\
\hline \multirow{4}{*}{$\begin{array}{l}\text { Cobertura flo- } \\
\text { restal do Brasil }\end{array}$} & Áreas de florestas naturais públicas e privadas por tipologia, biomas e UF \\
\hline & Áreas de florestas plantadas por espécies e UF \\
\hline & $\begin{array}{l}\text { Áreas de florestas com função de proteção e conservação do solo, água e biodiversidade } \\
\text { (florestas em UCs de proteção integral e de uso sustentável) }\end{array}$ \\
\hline & Áreas de florestas com função social (terras indígenas, Resex e RDS) \\
\hline \multirow{6}{*}{$\begin{array}{l}\text { Florestas de } \\
\text { produção }\end{array}$} & $\begin{array}{l}\text { Florestas naturais públicas e privadas (estoques e volumes comerciais dos manejos; produção de } \\
\text { madeira em tora; e de não madeireiros) por bioma/UF }\end{array}$ \\
\hline & $\begin{array}{l}\text { Florestas plantadas (estoques comerciais e produção de madeira em tora por espécies; e produção } \\
\text { de não madeireiros) por UF }\end{array}$ \\
\hline & Área anual de efetivo manejo florestal por bioma e UF \\
\hline & Área anual de plantio florestal por espécie e UF \\
\hline & Produção de lenha de florestas naturais e plantadas por UF \\
\hline & Resíduos da extração florestal (por tipo de floresta e atividade) por UF \\
\hline \multirow{4}{*}{$\begin{array}{l}\text { Propriedade das } \\
\text { terras florestais }\end{array}$} & Cadastro de florestas públicas \\
\hline & Cadastro de florestas privadas \\
\hline & Ato Declaratório Ambiental - ADA \\
\hline & Áreas de florestas sem dominialidade definida (terras devolutas) \\
\hline \multirow{6}{*}{$\begin{array}{l}\text { Indústria de } \\
\text { base florestal }\end{array}$} & $\begin{array}{l}\text { Capacidade instalada das indústrias de base florestal (serrarias, laminadoras, fábricas de painéis e } \\
\text { de celulose e papel) por UF }\end{array}$ \\
\hline & $\begin{array}{l}\text { Produção de serrados, laminados, compensados, painéis de madeira reconstituída (MDF, } \\
\text { aglomerados, chapa de fibra e OSB) e PMVA }\end{array}$ \\
\hline & Produção de celulose e papel por tipo e UF \\
\hline & $\begin{array}{l}\text { Capacidade instalada de produção de carvão vegetal (fornos e carvoarias das siderúrgicas à carvão } \\
\text { e produção de carvão independente) }\end{array}$ \\
\hline & Produção de carvão vegetal (florestas naturais e plantadas) por bioma e UF \\
\hline & Produção de não madeireiros (uso alimentar, industrial e medicinal) \\
\hline
\end{tabular}


Tabela 1. Continuação...

Table 1. Continue....

\section{MÓDULO \\ TEMÁTICO \\ PRINCIPAIS TIPOS DE INFORMAÇÕES FLORESTAIS}

Mercado interno de produtos madeireiros (consumo e preços de madeira em tora, serrados, compensados, laminados, PMVA, painéis, lenha e carvão vegetal)

Mercado de produtos

florestais

Tecnologia de
produtos
florestais

Biodiversidade florestal

Mudanças florestais e climáticas

Economia e estatística florestal

Legislação florestal

Certificação florestal

Ensino e pesquisa florestal
Mercado interno de produtos não madeireiros (consumo e preços de não madeireiros para uso alimentar, industrial, medicinal e ornamental)

Mercado externo de produtos madeireiros, não madeireiros, celulose e papel (quantidades exportadas, preços e destinos) por tipo de produto e UF

Fluxo de comércio interno de produtos florestais madeireiros e não madeireiros (origem e destino dos produtos)

Identificação e agrupamento de espécies florestais tropicais

Características gerais das madeiras brasileiras (cor, cheiro, grã, textura, figura, teor de extrativos, secagem e durabilidade natural)

Propriedades físicas (densidade, contração e inchamento volumétrico)

Propriedades mecânicas (módulo de elasticidade e tensões de ruptura a compressão, flexão, tração e cisalhamento)

Qualidade dos produtos madeireiros (trabalhabilidade, padronização, normalização e índice de rendimento no processamento primário)

Indicação dos melhores usos para as madeiras brasileiras

Catálogo das espécies arbóreas nativas (nomenclatura oficial)

Catálogo das espécies arbóreas plantadas (nomenclatura oficial)

Lista das espécies arbóreas ameaçadas de extinção (nomenclatura oficial, descrição e código de identificação das espécies)

Relação das parcelas permanentes (número, área, localização e dados)

Indicadores da biodiversidade florestal por bioma

Números do desmatamento (evolução histórica, taxa anual, área anual e as autorizações de desmatamento concedidas pelos governos federal e estadual)

Incêndios florestais (série histórica, focos de calor e áreas queimadas)

Áreas florestais degradadas ou alteradas, por bioma e UF

Emissões e estoques de carbono

PIB Florestal (Valor Bruto da Produção do setor florestal, geração de emprego e renda, recolhimento de tributos etc.)

Balança comercial de produtos florestais

Investimentos públicos e privados na área florestal (programados e realizados)

Crédito para a atividade florestal (programas, fomento e financiamentos)

Indicadores socioeconômicos da área florestal

Área anual de efetivo manejo florestal por bioma e UF

Área anual de plantio florestal por espécie e UF

Séries históricas (da evolução da produção de madeira em toras; das áreas de manejo florestal; das áreas de plantio de florestas; da produção, consumo, preço e exportação dos produtos; da balança comercial dos produtos florestais etc.)

Legislação sobre uso, proteção e conservação das florestas (leis, decretos, portarias e instruções normativas, tanto no âmbito federal quanto estadual)

Resoluções do Conama aplicadas à área florestal

Projetos de lei relacionados às florestas em tramitação no Congresso Nacional

Empresas e planos de manejo certificados (áreas, localização e volumes)

Empresas e áreas de florestas plantadas certificadas (localização e volumes)

Produtos madeireiros e não madeireiros certificados (cadeia de custódia)

Cursos de graduação e pós-graduação por área temática

Cursos técnicos e de treinamento por área temática

Projetos de pesquisa financiados com recursos públicos por área temática

Dissertações e teses na área florestal (consulta: bancos da CAPES, CNPq, USP)

Publicações técnico-científicas (livros, periódicos, trabalhos técnicos etc.) 
(professores, pesquisadores de instituições de ensino e pesquisa); e d) setor não governamental e outros (gestores, pesquisadores, consultores e técnicos vinculados a ONGs ou outros tipos de instituições).

\subsection{Coleta de dados}

A versão definitiva do questionário compôs-se de 58 perguntas para serem avaliadas pelos juízes, com notas, em grau de importância, na escala de 1 a 10, onde 1 (mínimo) era pouco importante e 10 (máximo), muito importante. $\mathrm{O}$ questionário respondido foi direcionado eletronicamente para um banco de dados configurado em Access, para facilitar a análise dos dados. O período de recebimento dos questionários foi entre os meses de outubro e dezembro de 2007.

\subsection{Análise dos dados}

Foi realizada uma análise estatística descritiva de todas as respostas do questionário, avaliando-se as medidas de frequência, de posição central e de dispersão (média, mediana, desvio padrão, valores mínimos e máximos, amplitude total, variância e coeficientes de variação). Semelhantemente a Almeida et al. (2009a), utilizou-se o teste de Duncan para uma análise comparativa das médias das notas atribuídas pelos juízes, admitindo-se nível de significância estatística de 5\%.

\section{RESULTADOS E DISCUSSÃO}

\subsection{Módulos temáticos e tipos de informação mais relevantes para o SIFLOR-BR}

Dos 160 questionários enviados, 72 foram respondidos e considerados válidos para a amostra deste trabalho, correspondendo a uma taxa de retorno de $45 \%$ do universo de juízes selecionados. De acordo com Malhotra (2001), esse percentual pode ser considerado alto, uma vez que taxa de retorno de $15 \%$ já seria considerada bom índice de retorno para pesquisas que não envolvem incentivos diretos (Antoni et al., 2006). Vasques et al. (2011) e Silva et al. (2010), analisando a estratégia das empresas de base florestal e utilizando metodologia semelhante, alcançaram retorno de $37,5 \%$ e $36,4 \%$ dos questionários aplicados, respectivamente.

A Tabela 2 mostra as estatísticas descritivas para os 12 módulos temáticos. As notas atribuídas indicam a dimensão que o SIFLOR-BR deve ter, na opinião dos juízes. De modo geral, as médias das notas de cada módulo temático (Tabela 2) podem ser consideradas elevadas - a média geral para todos os módulos foi de 8,67 . Isso demonstra que o comportamento dos juízes foi no sentido de tratar como importante todos os temas ou módulos temáticos propostos.

Os coeficientes de variação (Tabela 2) mostram uma dispersão reduzida para os valores em torno das médias de cada módulo, pois todos se registraram valores abaixo de 30\%, particularmente para módulos 2 (Florestas de produção), 4 (Indústria de base florestal), 5 (Mercado de produtos florestais), 6 (Tecnologia de produtos florestais) e 9 (Economia e estatística florestal), demonstrando uma concordância entre os especialistas. Segundo Pestana \& Gageiro (2005), pode-se avaliar a magnitude do coeficiente de variação $(\mathrm{CV})$ conforme a seguinte regra: $\mathrm{CV} \leq 15 \%$ = dispersão fraca; $15 \%<\mathrm{CV} \leq 30 \%$ = dispersão média; e CV > 30\% = dispersão elevada.

De acordo com os juízes, o módulo temático Florestas de produção é o mais importante, com média de 9,26. A Certificação florestal, por sua vez, com média de 7,72, é o módulo de menor importância. Esse módulo foi também o que apresentou o maior desvio padrão $(2,11)$ e o maior coeficiente de variação $(27,27 \%)$, indicando uma maior divergência na avaliação dos juízes. Os módulos 1 (Cobertura florestal do Brasil), 3 (Propriedade das terras florestais), 7 (Biodiversidade florestal), 8 (Mudanças florestais) e 10 (Legislação florestal) também apresentaram intervalos maiores entre os valores mínimos e máximos, sugerindo uma maior divergência, na avaliação dos juízes, entre as variáveis analisadas.

Conforme os resultados do teste de Duncan, pode-se verificar a existência de diferença estatisticamente significativa entre as médias. A interpretação do teste é que as médias dentro de cada grupo são estatisticamente iguais ao nível de 5\% (Tabela 3).

A partir dos resultados do teste de Duncan pode-se então propor uma classificação das variáveis em três conjuntos, conforme o grau de importância atribuído pelos juízes, separando-se as variáveis em graus de importância alta, média e baixa (Tabela 4). Identificou-se a variável referente à Certificação florestal como menos importante, o grupo de variáveis encabeçado pelo módulo Florestas de produção como o mais importante e as outras variáveis dentro de um grupo com de importância intermediária (Tabela 4). 
Tabela 2. Estatísticas descritivas dos módulos temáticos propostos para o SIFLOR-BR.

Table 2. Descriptive statistics of thematic modules proposed for the SIFLOR-BR.

\begin{tabular}{|lcccccc|}
\hline \multicolumn{1}{c}{ Módulos temáticos } & Média & Mediana & $\begin{array}{c}\text { Desvio } \\
\text { padrão }\end{array}$ & $\begin{array}{c}\text { Valor } \\
\text { mínimo }\end{array}$ & $\begin{array}{c}\text { Valor } \\
\text { máximo }\end{array}$ & $\begin{array}{c}\text { Coef. de } \\
\text { variação em \% }\end{array}$ \\
\hline 1 Cobertura florestal do Brasil & 9,00 & 10,0 & 1,74 & 1 & 10 & 19,37 \\
\hline 2 Florestas de produção & 9,26 & 10,0 & 1,17 & 5 & 10 & 12,68 \\
\hline 3 Propriedade das terras florestais & 8,40 & 9,0 & 2,04 & 1 & 10 & 24,27 \\
\hline 4 Indústria de base florestal & 8,82 & 9,0 & 1,40 & 5 & 10 & 15,83 \\
\hline 5 Mercado de produtos florestais & 8,99 & 10,0 & 1,51 & 2 & 10 & 16,75 \\
\hline 6 Tecnologia de produtos florestais & 8,68 & 9,0 & 1,44 & 4 & 10 & 16,61 \\
\hline 7 Biodiversidade florestal & 8,68 & 9,0 & 1,64 & 1 & 10 & 18,85 \\
\hline 8 Mudanças florestais & 8,31 & 9,0 & 1,76 & 1 & 10 & 21,14 \\
\hline 9 Economia e estatística florestal & 9,07 & 10,0 & 1,37 & 3 & 10 & 15,07 \\
\hline 10 Legislação florestal & 8,58 & 9,0 & 1,81 & 1 & 10 & 21,04 \\
\hline 11 Certificação florestal & 7,72 & 8,0 & 2,11 & 1 & 10 & 27,27 \\
\hline 12 Ensino e pesquisa florestal & 8,53 & 9,0 & 1,59 & 3 & 10 & 18,66 \\
\hline
\end{tabular}

Tabela 3. Resultados do teste de Duncan.

Table 3. Duncan Test results.

\begin{tabular}{|c|c|c|c|c|c|}
\hline \multirow{2}{*}{ Módulos temáticos } & \multicolumn{5}{|c|}{ Grupos } \\
\hline & 1 & 2 & 3 & 4 & 5 \\
\hline Certificação florestal & 7,72 & & & & \\
\hline Mudanças florestais & & 8,31 & & & \\
\hline Propriedade das terras & & 8,40 & 8,40 & & \\
\hline Ensino e pesquisa & & 8,53 & 8,53 & 8,53 & \\
\hline Legislação florestal & & 8,58 & 8,58 & 8,58 & \\
\hline Biodiversidade florestal & & 8,68 & 8,68 & 8,68 & 8,68 \\
\hline Tecnologia de produtos & & 8,68 & 8,68 & 8,68 & 8,68 \\
\hline Indústria florestal & & 8,82 & 8,82 & 8,82 & 8,82 \\
\hline Mercado de produtos & & & 8,99 & 8,99 & 8,99 \\
\hline Cobertura florestal & & & 9,03 & 9,03 & 9,03 \\
\hline Economia e estatística & & & & 9,07 & 9,07 \\
\hline Florestas de produção & & & & & 9,26 \\
\hline
\end{tabular}

Tabela 4. Agrupamento dos módulos temáticos por ordem de importância.

Table 4. Grouping of thematic modules in order of importance.

\section{IMPORTÂNCIA}

Alta

\section{MÓDULOS TEMÁTICOS}

Florestas de produção

Economia e estatística florestal

Cobertura florestal no Brasil

Mercado de produtos florestais

Indústria de base florestal

Tecnologia de produtos florestais

Biodiversidade florestal

Legislação florestal

Ensino e pesquisa florestal

Propriedade das terras florestais

Mudanças florestais e climáticas

Baixa

Certificação florestal
MÉDIA

9,26

9,07

9,00

8,99

8,82

8,68

8,68

8,58

8,53

8,40

8,31

7,72 
Na percepção dos juízes, o SIFLOR-BR deve abordar, prioritariamente, as variáveis de maior importância: Florestas de produção; Economia e estatística florestal, Cobertura florestal no Brasil; Mercado de produtos florestais, Indústria de base florestal; Tecnologia de produtos florestais; e Biodiversidade florestal.

Aparentemente, estiveram alheias à percepção dos juízes a disponibilidade de dados florestais de algumas fontes, no Brasil, provavelmente devido as limitações dessas fontes na divulgação das informações. O IBGE, Instituto Brasileiro de Geografia e Estatística, por meio das suas pesquisas sobre silvicultura e extração vegetal, fornece dados sobre valores e volumes produzidos de bens madeireiros e não madeireiros, bem como, por intermédio da sua pesquisa industrial, disponibiliza informações sobre as indústrias de base florestal, as quais estão entre as principais informações destacadas pelos juízes para a composição do SIFLOR-BR. Nesse caso, a percepção dos juízes pode ter sido influenciada pelo fato de os dados do IBGE serem incompletos e agregados. Com todas as limitações, destaca-se a importância das informações fornecido pelas pesquisas do IBGE sobre a extração vegetal e silvicultura, podendo-se citar vários trabalhos que utilizaram tais dados como, por exemplo: Almeida et al. (2009b, c; 2010a, b) e Angelo et al. (2009), em análises do mercado e da indústria de base florestal.

Informações mais específicas relacionadas à produção florestal são disponibilizadas por algumas associações como, por exemplo: Abraf (Associação Brasileira de Produtores de Florestas Plantadas), Abimci (Associação Brasileira da Indústria da Madeira Processada Mecanicamente), Bracelpa (Associação Brasileira de Celulose e Papel), Abipa (Associação Brasileira da Indústria de Painéis de Madeira), entre outras. Porém, tal como os dados fornecidos pelo IBGE, a informação dessas fontes também apresenta algumas limitações, principalmente de ordem estatística como, por exemplo: descontinuidade das séries de dados, amostragens insuficientes, entre outras. Além disso, a coleta desses dados depende da força dos respectivos setores, destacando-se a abundância de informações do setor de papel e celulose e a carência de dados sobre a atividade florestal na Amazônia.

Além das associações, outros órgãos fornecem informações florestais, porém em caráter regional, destacando-se: o Cepea (Centro de Estudos Avançados em
Economia Aplicada) para o Estado de São Paulo, a Seab (Secretaria da Agricultura e Abastecimento do Paraná) e a Cepa (Centro de Estudos de Safras e Mercados), em Santa Catarina, porém não são todos os estados que possuem a mesma estrutura de informação e não existe padrão na coleta de dados, o que impossibilita comparações e análises mais específicas.

Diante das fontes disponíveis de dados voltados para a produção florestal, observa-se algumas sobreposições e falta de informações, além de falta de padronização, organização, rigor estatístico, acessibilidade, entre outros problemas, que acabam tornando as informações inacessíveis ou pouco úteis para uma série de análises, contribuindo para entender a percepção dos juízes em relação às principais informações que devem compor o SIFLOR-BR.

Observa-se ainda que alguns temas em evidência como biodiversidade, mudanças florestais e a certificação tiveram avaliações distintas, sendo que a certificação chama mais atenção por ser considerada aquela de menor importância na avaliação dos juízes. Essas informações, no Brasil, são normalmente disponibilizadas pelo Ibama (Instituto Brasileiro do Meio Ambiente e dos Recursos Naturais Renováveis) e por órgãos ambientais estaduais e municipais, que apresentam limitações para compilação e coleta de dados e excessiva burocracia para a sua obtenção, o que dificulta a difusão dessas informações para a sociedade em geral.

\subsection{As implicações deste estudo na construção da proposta do SIFLOR-BR}

Considerando a complexidade dos dados e das informações florestais, a diversidade de fontes, bem como a heterogeneidade de padrões e da suas formas de apresentação, pode-se dizer que os seus resultados, em geral, são influenciados por um número grande de elementos e variáveis. Portanto, saber selecionar, eleger e conhecer as características dos dados a serem coletados, ordenados, atualizados e disseminados pelo sistema de gestão dos recursos florestais e ambientais é o grande desafio para qualquer modelo de sistema nessa área de trabalho.

A proposta de construção do SIFLOR-BR parte da identificação de alguns problemas e dificuldades com que tanto a formulação de políticas públicas quanto a gestão dos recursos florestais convivem há décadas, sem soluções. E essas, quando surgem, são, 
em geral, emergenciais, pontuais ou sofrem de solução de continuidade. O país não dispõe, ainda, de um sistema institucionalizado de informações florestais como instrumento de conhecimento permanente das realidades florestais, que auxilie no planejamento estratégico do uso racional e da conservação desses recursos.

Um setor que não dispõe de informações não consegue planejar, avaliar cenários e tendências, nem mesmo fazer correções de rumo em suas políticas e decisões estratégicas. Portanto, um Sistema de Informação como instrumento estratégico para a gestão florestal é fundamental, urgente e imprescindível ao planejamento e ao processo de tomada de decisão nas instituições públicas e privadas da área florestal.

\section{CONCLUSÕES}

Apesar de os juízes terem considerado todos os módulos temáticos importantes, em função das notas que receberam, a análise dos resultados indica uma priorização por informações nos temas Produção e Economia e estatística florestal.

A consideração geral deste estudo é de que a construção do SIFLOR-BR é possível e fundamental. Para tanto, um primeiro passo é a identificação dos principais elementos necessários à implementação desse importante e indispensável instrumento para a gestão estratégica dos recursos florestais brasileiros. Entretanto, se políticas e ações no sentido de viabilizar a construção e consolidação do sistema proposto ou semelhante não forem adotadas, a gestão florestal no Brasil continuará convivendo com a falta de informações sistematizadas e com dados desatualizados e de qualidade duvidosa.

\section{STATUS DA SUBMISSÃO}

Recebido: 18 jul., 2012

Aceito: 26 jun., 2016

\section{AUTOR(ES) PARA CORRESPONDÊNCIA}

\author{
Alexandre Nascimento de Almeida \\ Faculdade UnB de Planaltina - FUP, \\ Universidade de Brasília - UnB, Área \\ Universitária, 1, Vila Nossa Senhora de Fátima, \\ CEP 73300-000, Planaltina, DF, Brasil \\ e-mail: alexalmeida@unb.br
}

\section{REFERENNCIAS}

Albertin AL. Aumentando as chances de sucesso no desenvolvimento e implementação de sistemas de informação. Revista de Administração de Empresas 1996; 36(3): 61-69. http://dx.doi.org/10.1590/S0034-75901996000300008.

Almeida NA, Angelo H, Silva JCGL, Hoeflich VA. Mercado de madeiras tropicais: substituição na demanda de exportação. Acta Amazonica 2010a; 40(1): 119-126. http://dx.doi.org/10.1590/S0044-59672010000100015.

Almeida NA, Angelo H, Silva JCGL, Nuñez BHC. Análise econométrica do mercado de madeira em tora para o processamento mecânico no Estado do Paraná. Scientia Forestalis 2009b; 37(84): 377-386.

Almeida NA, Bittencourt AM, Santos AJ, Eisfeld CL, Souza VS. Evolução da produção e preço dos principais produtos florestais não-madeireiros extrativos do Brasil. Cerne 2009c; 15(3): 282-287.

Almeida NA, Loper AA, Silva JCGL, Hoeflich VA. Modelo estratégico do pólo moveleiro do Alto Vale do Rio Negro sob os conceitos de Porter. Scientia Forestalis 2009a; 37(83): 233-242.

Almeida NA, Silva JCGL, Ângelo H, Nuñez BHC. Análise dos fatores que influenciam o preço da madeira em tora para o processamento mecânico no Paraná. Cerne 2010b; 16(2): 243-250. http://dx.doi.org/10.1590/S010477602010000200017.

Angelo H, Almeida NA, Serrano ALM. Determinantes da demanda de madeira em toras para celulose no Brasil. Scientia Forestalis 2009; 37(84): 491-498.

Antoni VL, Damacena C, Lezana AGR. Um modelo preditivo de orientação para o mercado: um estudo no contexto do ensino superior brasileiro. Teoria e Evidência Econômica 2006; 14: 143-165.

Beattie WD, Nascimento JR. Sistema de Informações do Setor Florestal do Brasil-SISF: situação atual e plano de trabalho. Brasília: COPLAN/IBDF/MA; 1979.

Brasil. Ministério do Meio Ambiente - MMA. Exposição de motivos do anteprojeto de lei que dispõe sobre a gestão de florestas públicas. Brasília: MMA/GM; 2005. 8 p.

Brena DA. Inventário Florestal Nacional: Proposta de um Sistema para o Brasil [tese]. Curitiba: Escola de Florestas, Universidade Federal do Paraná; 1995.

Campos MP Fo. Os sistemas de informação e as modernas tendências da tecnologia e dos negócios. Revista de Administração de Empresas 1994; 34(6): 33-45. http:// dx.doi.org/10.1590/S0034-75901994000600005.

Davis FD. Perceived usefulness, perceived case of use, and user acceptance of information technology. Management Information Systems Quarterly 1989; 13(3): 983-1002. http://dx.doi.org/10.2307/249008. 
Graça LR, Mendes JB, Wiecheteck M. Sistema de Informações Florestais e Ambientais. Curitiba: MMA/PNF; 2002. 26 p. Pré-projeto.

Lachtermacher G. Pesquisa operacional na tomada de decisões. 4. ed. Rio de Janeiro: Elsevier; 2002. 144 p.

Laudon KC, Laudon JP. Sistemas de Informações Gerenciais: administrando a empresa digital. 5. ed. São Paulo: Pearson/ Prentice Hall; 2004. 562 p.

Malhotra NK. Pesquisa de marketing: uma orientação aplicada. 3. ed. Porto Alegre: Bookman; 2001.

Nobre SR, Rodriguez LCE, Assis MLR, Amaral TM. Avaliação do uso da tecnologia da informação nos sistemas de administração da produção florestal. In: Anais do $8^{\circ}$ Congresso Florestal Brasileiro: Informações gerais e Trabalhos Voluntários; 2003; São Paulo. São Paulo: SBEF, SBS; 2003. 12 p.

Pestana MH, Gageiro JN. Análise de dados para ciências sociais: a complementariedade do SPSS. Lisboa: Edições Silabo; 2005.

Rezende DA. Alinhamento estratégico da tecnologia da informação ao planejamento estratégico: proposta de um modelo de estágios para governança em serviços públicos. Revista de Administração Pública 2004; 38(4): 519-542.
Rodrigues J Fo, Ludmer G. Sistema de Informação: que ciência é essa? Revista Gestão da Tecnologia e Sistemas de Informação 2005; 2(2): 151-156.

Serviço Florestal Brasileiro - SFB. Florestas do Brasil em resumo: 2010. Brasília: MMA; 2010. [citado em 2012 out. 22]. Disponível em: http://www.florestal.gov.br

Silva JCGL, Almeida AN. Modelo de estratégias de marketing internacional. Revista Forestal Latinoamericana 2009; 24(2): 30-45.

Silva JCGL, Almeida AN, Hildebrand E, Sawinski J Jr, Lacowicz PG. Estratégias de posicionamento no mercado internacional das empresas brasileiras de papel e celulose. Floresta 2010; 40(4): 801-812. http://dx.doi.org/10.5380/ rf.v40i4.20331.

Soares JS Jr, Quintella RH. Descoberta de conhecimento em bases de dados públicas: uma proposta de estruturação metodológica. Revista da Administração Pública 2005; 39(5): 1077-1107.

Vasques AG, Silva JCGL, Almeida AN. A identificação da orientação estratégica da empresa florestal no Brasil: uma aplicação da teoria de Porter. Floresta 2011; 41(4): 695-706. http://dx.doi.org/10.5380/rf.v41i4.25335. 